Revue d'histoire du XIXe siècle

Société d'histoire de la révolution de 1848 et des

révolutions du XIXe siècle

Religion, politique et culture au XIXe siècle

\title{
Les catholiques libéraux en révolution avant l'heure. Fin 1847 : Suisse - Italie - France
}

Sylvain Milbach

\section{(2) OpenEdition \\ Journals}

Édition électronique

URL : http://journals.openedition.org/rh19/617

DOI : $10.4000 /$ rh 19.617

ISSN : $1777-5329$

Éditeur

La Société de 1848

Édition imprimée

Date de publication : 1 juin 2004

Pagination : $59-78$

ISSN : 1265-1354

Référence électronique

Sylvain Milbach, «Les catholiques libéraux en révolution avant l'heure. Fin 1847 : Suisse - Italie -

France », Revue d'histoire du XIXe siècle [En ligne], 28| 2004, mis en ligne le 07 avril 2008, consulté le 03 mai 2019. URL : http://journals.openedition.org/rh19/617 ; DOI : 10.4000/rh19.617

Ce document a été généré automatiquement le 3 mai 2019.

Tous droits réservés 


\title{
Les catholiques libéraux en révolution avant l'heure. Fin 1847 : Suisse - Italie - France
}

\author{
Sylvain Milbach
}

15 novembre 1847. Le comte de Montalembert, en villégiature dans son château de La Roche-en-Brenil (Côte-d'Or), rédige son testament. Le 11, après que sa femme l'en ait une première fois dissuadé, il prend la route de nuit pour Dijon où l'attend la diligence qui le mènera à Besançon. De là, il franchira la frontière suisse pour atteindre Fribourg et s'enrôler sous le drapeau du Sonderbund. «Mais, chose étrange et déplorable, à mesure que j'approche de cette frontière suisse, où sont concentrées depuis si longtemps les plus vives émotions de mon âme, je sens mon ardeur décroître et ma résolution fléchir " ${ }^{1}$. Le 12 novembre au matin, après un voyage nocturne, le voici de retour à Dijon. « Je dors la plus grande partie de la nuit et en arrivant à Dijon, je me révolte de ma décision d'hier soir. Je suis fortement tenté de revenir sur elle et de retourner à Besançon. Mais la triste conviction d'arriver trop tard me domine et m'arrête. Je sens que j'ai fait la plus sotte campagne qu'il soit possible d'imaginer " 2. L'imaginaire de croisade passé à l'épreuve du réalisme politique, Montalembert retrouve sa demeure de La Roche-en-Brenil. Il interviendra "parlementairement» pour la cause des catholiques suisses, et non militairement : la croisade s'effectuera du haut de la tribune des pairs.

2 Janvier 1848. À Paris, Montalembert est à nouveau en proie au doute : doit-il monter à la tribune pour Pie IX? Il écoute les voix de ses proches, ceux qui, quelques semaines auparavant, s'accordaient pour lui recommander de remettre sur le métier son Monasticon (futur Moines d'Occident). L'abbé Dupanloup prône l'abstention. L'autre voix, Théophile Foisset, s'emporte et emporte la décision: "Mon ami, décidément M. Dupanloup ne m'arrête pas: M. Dupanloup n'est pas, que je sache, un homme politique, il ne fait pas autorité ici ${ }^{3}$. Foisset affirme donc que la question italienne n'est pas l'affaire des hommes d'Église: point d'exclusive ici. C'est une question politique, une question où l'action laïque a un droit prééminent de célébration. La cause est commune, l'affaire est 
entendue : elle est religieuse, au-dessus des partis. Mais, pour servir cette cause, il faut s'appuyer sur les ressorts modernes de l'action politique, étrangers à la culture ecclésiastique que souvent les deux amis décrivent comme ancrée dans des postures passéistes et dont ils fustigent la timidité face aux réalités contemporaines. Ce jugement n'épargne pas même ici l'abbé Dupanloup, pourtant si estimé, parce qu'il reste alors l'homme des discrètes et diplomatiques négociations.

3 Au tournant de l'année 1847, le "mouvement catholique ${ }^{4}$ est aux prises avec ses contradictions. Celles-ci ne sont pas nouvelles mais, pour ainsi dire, inhérentes à la nature de la dynamique impulsée depuis 1843, date de constitution du Comité de défense de la liberté religieuse qui se fixait pour objectif d'obtenir la liberté de l'enseignement secondaire. Toutefois, les événements internationaux vont les révéler et préparer les divisions que va connaitre le mouvement en 1848 , car la distinction entre la prise de position politique et la défense de la religion devient par trop artificielle. La présente contribution s'efforcera de suivre l'élaboration des deux discours sur les affaires de Suisse et d'Italie à l'aide des propres archives de Montalembert et de celles de Foisset, conseiller occulte du fougueux comte.

4 En quelques lignes, plantons le décor. L'année 1847 fut une année bien remplie pour le mouvement catholique. Il y a bien entendu l'action proprement dite du Comité de défense de la liberté religieuse (devenu Comité électoral pour la défense des intérêts religieux en 1844) : mobiliser les troupes en vue des élections, pétitions et débats. À l'automne, Montalembert prévoit d'épauler H. de Riancey (secrétaire et « commis-voyageur » dudit Comité) dans ses voyages en province : il s'agit de recruter et rasséréner les troupes (tout spécialement l'épiscopat). En juillet, Montalembert a publié une brochure contre le projet Liardières, du nom du rapporteur de la commission de la Chambre des députés, avantdernier avatar de la lutte pour la liberté de l'enseignement secondaire. Ce projet, mort-né comme les précédents (projets Villemain de 1841 et 1844), inquiète nos hommes. Déformant le projet du ministre Salvandy pour une « libéralisation » de l'enseignement secondaire, il sera en fait emporté sans discussion par la révolution de 1848. Durant cette année 1847, le comte est monté plusieurs fois à la tribune au sujet de la Pologne opprimée ${ }^{5}$. Ces interventions, indiscutablement brillantes, lui valent un grand succès à la Chambre des Pairs. Il est au sommet de sa gloire oratoire et passe pour un défenseur des nationalités et des peuples opprimés. De l'autre main, l'infatigable comte rédige ses Moines d'Occident qui, comme on le sait, visent à revaloriser l'action civilisatrice des ordres religieux, sur fond d'idéalisation du Moyen Âge.

Quoique plus modestement, Foisset n'est pas inactif ${ }^{6}$. Il est entré en polémique avec le protestantisme. Son ouvrage connaît, au sein du cénacle catholique libéral, un durable succès d'estime ${ }^{7}$. Il poursuit son action de relais du Comité de défense au sein de la Bourgogne et se livre, plus ou moins anonymement, à son apostolat, si précieux à ses yeux car si proprement "catholique libéral», de publiciste. En Bourgogne, il patronne officieusement le Spectateur (que Montalembert finance en partie), une manière d'Univers local, plus «orthodoxe» que le premier puisqu'à cette date Montalembert est pour le moins en froid avec Veuillot ${ }^{8}$. Surtout, il joue un rôle central dans le Correspondant. Il y avait vivement attaqué l'Université en la personne de Cousin. En cette fin d'année 1847, il publie notamment un long compte-rendu sur l'Histoire des Girondins de Lamartine, ouvrage dans lequel il perçoit la réhabilitation de ce qu'il nomme le radicalisme.

En somme, on le voit, diverses actions auxquelles les événements internationaux de cette fin de 1847 vont donner un net regain d'actualité. Nos hommes voient dès lors dans ces 
événements la confirmation de leur activité. Ils voient se dessiner ce sens de l'histoire qu'ils redoutent tant. Tout d'abord en Suisse, où les tensions s'accentuent depuis 1845. Ensuite, en Italie avec les événements romains. En France aussi, où débute la campagne des banquets.

En Suisse, ces tensions s'inscrivent dans le cadre de ce que les historiens suisses nomment la « régénération », c'est-à-dire une volonté née au début des années 1830 de révision du Pacte fédéral de 1815 dans le sens d'un État fédératif ${ }^{9}$. Bien des cantons sont frappés par une forte instabilité politique, qui amène des réaménagements constitutionnels à répétition. Conservateurs et libéraux s'affrontent. Cette longue période de tension est officialisée en 1845 par la création du Sonderbund, ligue défensive des cantons catholiques, qui constitue aux yeux des cantons radicaux, une violation du Pacte de 1815. En mai 1847, les radicaux sont majoritaires à la Diète et réclament la dissolution de la ligue. Les hostilités se déroulent durant le mois de novembre 1847 et aboutissent à une défaite sans appel des sept cantons. Nos observateurs s'inscrivent à rebours des conclusions des historiens actuels de la Suisse: ils percevront la crise du Sonderbund comme fondamentalement religieuse, toute tentative de « centralisation » étant par essence à la fois liberticide et jacobine, c'est-à-dire antireligieuse.

8 À Rome, l'avènement de Pie IX (juin 1846) semble plutôt aller dans le sens de ce qu'attend le mouvement catholique. Les premières mesures du nouveau souverain pontife (amnistie pour les prisonniers politiques, assouplissement de la censure...) tranchent avec le règne de Grégoire XVI. En 1847, les réformes institutionnelles (Consulta, municipalité romaine...) semblent présager une évolution libérale. Depuis les travaux de Roger Aubert, on sait l'illusion, ou le malentendu, que suscitèrent les premiers pas politiques de Pie IX. Nos observateurs ne la partagèrent jamais vraiment. Ce qui les inquiéta définitivement, c'est la fièvre nationale qui agita les rues de Rome dans les derniers mois de 1847. Derrière les demandes libérales, se cachait le prurit des factions; au cœur de l'Église divisée, se profilait la paralysie; sous les huées anti-autrichiennes, couvait l'inflammation unitaire. Ce n'était donc plus une libéralisation mais les symptômes d'une révolution. Tel fut le diagnostic de Montalembert et Foisset qui regardaient aussi la France en ce mois de décembre 1847.

10

9 Dans l'approche des soubresauts européens de cette fin d'année 1847, on ne peut faire l'économie de la question des jésuites. On sait que leur arrivée à Lucerne, à l'appel de la ville, est un des éléments qui déclenchent les hostilités qui déboucheront sur la guerre éclair de novembre. En Italie, et en lien avec les événements suisses, Gioberti, auréolé de son Primato ${ }^{11}$ qui apparaît alors comme une prophétie en voie de réalisation, lance un pamphlet de renom contre la Compagnie : Il Gesuita moderno, agrémenté dans sa seconde édition (fin 1847) d'une virulente réponse aux attaques ${ }^{12}$. L'auteur contribue surtout à soulever l'opinion romaine contre les jésuites, assimilés à la défense des intérêts conservateurs, tant en Italie qu'en Suisse, donc au " parti autrichien ». Si les catholiques libéraux français pouvaient se féliciter de cette dissociation des intérêts du Saint-Siège de ceux de l'Autriche, ils s'inquiètent en revanche des germes de division que jette Gioberti. Ce qui lui est reproché, c'est surtout une attaque publique qui fragilise le Pape : Gioberti incarne les passions du siècle qui s'insinuent dans l'Église alors que, somme toute, les jésuites incarnent l'obéissance.

10 En fait, les jésuites divisent les membres du mouvement catholique. S'ils rejettent l'attitude de Gioberti, ils considèrent bel et bien que les fils de saint Ignace incarnent l'aile 
réactionnaire de l'Église : il ne fait nul doute qu'ils constituent un frein aux réformes de Pie IX. Montalembert lui-même le sait bien quand il écrit à Dupanloup pour qu'il pousse le P. Ravignan ${ }^{13}$ à aller à Rome "y inspirer de la confiance au Pape et la véritable intelligence de la situation au général de la Compagnie » ${ }^{14}$. Ce manque "d'intelligence de la situation ", Foisset le reprochera bientôt plus vertement : il estimera que les jésuites auraient dû se retirer de Lucerne afin de délégitimer l'action de la Diète. Il les rend donc, dans une certaine mesure, responsables de la tournure des événements. Plus généralement, Foisset reproche à l'ordre d'endosser le rôle que les adversaires de l'Église veulent lui faire jouer, tant en France qu'outre-monts : ils servent l'assimilation de l'Église aux forces passéistes.

11 Montalembert avait déjà pris publiquement, lors de ses discours à la Chambre des pairs de 1844 et 1845 , la défense des jésuites. Il assumait alors les conséquences de la campagne lancée pour la liberté de l'enseignement qui avait réveillé le mythe de l'ordre noir ${ }^{15}$ et avait abouti aux célèbres répliques de Michelet et Quinet, sans oublier le succès du Juif errant de Sue où Montalembert était personnellement caricaturé. Le mouvement catholique n'avait affiché qu'une solidarité de façade : à la méfiance envers les jésuites, s'ajoutait la menace que leur réputation faisait désormais planer sur la cause du Comité.

En 1847, l'instrumentalisation des jésuites en Suisse et en Italie soulève à nouveau la sainte colère du comte. Il est décidé, une fois encore, à aller pourfendre à la tribune les ennemis de l'ordre. Comme en 1844, il se heurte à la "timidité » de l'équipe du Correspondant et aux réticences de Foisset. Celui-ci lui rappelle les conseils qu'il lui avait quatre ans plus tôt prodigués et la justesse de son calcul : l'épouvantail jésuite avait été agité par les défenseurs du monopole universitaire afin de faire capoter le débat sur la liberté d'enseignement, « vous m'avez envoyé promener, vous savez le reste » ${ }^{16}$. En cette fin d'année 1847, bien que les enjeux ne soient pas les mêmes, la question des jésuites n'en est pas moins un piège. Aussi prie-t-il le comte de s'abstenir, dans ses discours à venir, d'aborder ce sujet.

L'échange épistolaire n'est pas moins vif qu'en 1844. Le magistrat est sommé de se justifier et le comte dresse à son encontre l'accusation de "giobertisme». Foisset confesse n'avoir pas aimé les jésuites, trop compromis sous le ministère Polignac. Mais il rappelle également, qu'il avait pris leur défense lors des ordonnances de 1828 qui prescrivaient leur proscription de l'enseignement. Le magistrat n'est guère à l'aise ici : il cherche à contourner les suspicions d'un Montalembert particulièrement ombrageux sur ce point. Aussi, rappelle-t-il que le P. Roothan, le général de la Compagnie, a qualifié le P. Ventura, après son éloge d'O'Connell, de démagogue. Dans ce discours de juin 1846, le P. Ventura honorait la mémoire du héros émancipateur de l'Irlande catholique, figure tutélaire du mouvement catholique depuis l'Avenir. Ce discours marquait, pouvait-on penser, une inflexion des positions romaines à l'égard de l'agir catholique dans le monde contemporain et, notamment, à l'égard de l'action politique laïque. Or, pour Foisset, Ventura s'est situé bien en-deçà des positions du mouvement catholique. Dans ce cas, "Que dirait-il [Roothan] de nous ! " ${ }^{17}$

De là, il suggère à Montalembert que les jésuites adoptent des positions que le mouvement catholique s'efforce de désarmer, qu'en somme on ne saurait faire cause commune et il lui échappe : "naturellement, ils n'inclinent pas aux réformes». Mais il fait un pas en arrière, de manière à disqualifier toute accusation de « jésuitophobie », en s'appuyant sur les propos de Lacordaire de retour de Rome: il semble avéré que les attaques ne sont que d'honteuses calomnies (mais là n'est pas le problème) et que le 
P. Roothan est « en voie de changement » vers une attitude plus conciliatrice à l'égard des réalités contemporaines. En fait, la dernière lettre de Lacordaire à Foisset ne dit rien de cela et, pour tout dire, résume assez parfaitement l'opinion de Foisset : les jésuites sont rétrogrades, ils ont accumulé erreur sur erreur depuis 1815, il faut désolidariser la cause de l'Église de celle de l'ordre, il n'est rien à faire pour eux ${ }^{18}$. Le but de la manœuvre de Foisset est naturellement de se défendre de toute accusation de parti pris et de faire glisser l'argumentation vers des aspects strictement pragmatiques: ne prêtons pas le flanc aux attaques et sachons bien que « les jésuites sont le défaut de notre cuirasse ». En somme, toute défense des intérêts de la religion doit écarter la question des jésuites.

Foisset n'est pas seul à mener cette lutte stratégique. Si Lacordaire n'intervient pas dans la préparation des discours, le P. Ravignan lui-même, demande à Montalembert de ne pas aborder la question de l'ordre à la tribune ${ }^{19}$. Il obéit par là aux directives du P. Roothan et de Pie IX : faisons le gros dos tandis que passe la tempête. De fait, à la tribune, la cause des jésuites est estompée. Au début du discours sur la Suisse, le comte, en esquivant la question, se paie le luxe de provoquer l'hilarité : « Je compte, pour ma part, ne pas dire un mot ni des traités, ni des dépêches, ni même des jésuites (On rit) ${ }^{20}$. C'est assez dire qu'on l'y attendait.

defaut de la cuirasse dont parle Foisset est précisément l'assimilation d'une posture de défense des intérêts religieux à celle d'une défense d'intérêts partisans. Or, les discours de 1847, on s'en rappelle, doivent être politiques, c'est-à-dire qu'ils doivent rallier les conservateurs de la Monarchie de Juillet. Si les jésuites sont repoussés, c'est parce qu'ils sont la mauvaise carte du conservatisme, l'assimilation aux régimes rétrogrades, et, qui plus est et très concrètement, le ferment, par réaction, de la révolution: les défendre, c'est faire le jeu de l'ennemi. Le réalisme politique impose de se désolidariser, non pas d'une vision théocratique qui reste en fin de compte le modèle latent de nos hommes, mais d'une attitude contre-productive. En somme, disons-le ainsi pour l'instant, une "théocratie modérée ", mâtinée d'un parlementarisme imposé par le sens de l'histoire, reste l'idéal bien contradictoire du mouvement catholique. C'est ce qui explique l'engouement premier pour les réformes de Pie IX, idéal d'une société libéralisée au nom de Dieu qui revalorise la prééminence morale du chef de l'Église et le rôle contemporain de la religion.

Dans ce contexte, comment expliquer l'obstination de Montalembert à défendre ouvertement les jésuites? On pourra invoquer des ressorts affectifs ainsi que des liens de fidélité : lors de la campagne de 1843-1845 pour l'expulsion de l'ordre, le comte noue des relations durables avec les membres les plus distingués de la Compagnie. La «trahison » de Rome affectera profondément Montalembert ${ }^{21}$. On pourra encore faire appel à l'imaginaire du comte, nourri d'une culture historique à l'œuvre dans le Monasticon en cours de rédaction : le sentiment de se croiser, la consolation d'être seul contre tous ou la gloire de lutter à contre-courant, la conviction de perpétuer un sens de l'honneur et le refus des compromissions modernes, inclinant toutes à la lâcheté (sentiment qui revient si souvent dans la correspondance et qui n'est pourtant que l'expression du parlementarisme si ardemment défendu). En somme, un sentiment aristocratique que Montalembert incarne avec brio. Mais ce portrait vaguement psychologique, ce don quichottisme, est trop peu rationnel pour expliquer l'attitude d'un homme rompu aux joutes politiques. En effet, si Montalembert veut défendre avec tant d'opiniâtreté les jésuites, c'est qu'il n'oublie pas le vrai combat du mouvement catholique que les événements viennent troubler. À l'instar de Foisset, il tient en piètre estime le clergé 
séculier et, dans la perspective d'une liberté de l'enseignement secondaire, il l'estime inapte à remplir une tâche d'enseignement. Ici, seuls les jésuites seraient à la hauteur de cette tâche. Lâcher les jésuites, c'est hypothéquer un enseignement catholique solide. Montalembert considère, à la lumière de ses études en cours sur les Moines d'Occident, que les ordres religieux ont toujours été le fer de lance de l'Église : les attaquer, c'est s'en prendre aux forces vives de l'Église. Il ne faut pas oublier qu'un des volets des libertés religieuses réclamées sous la Monarchie de Juillet est précisément celle des ordres religieux. Parmi ceux-ci, les jésuites ont mission d'enseignement secondaire. C'est sans doute l'argument le plus déterminant pour expliquer la volonté de défendre l'ordre. Les événements politiques d'outre-monts obligent à un repositionnement pragmatique : «Se conduire dans ce monde par des règles absolues, c'est un jeu sûr pour se perdre. L'absolu n'est pas de ce monde. Si l'on veut agir comme si la vérité absolue régnait sur la terre, on se casse le cou en pure perte. C'est le possible qu'il faut chercher, non pas le mieux absolu mais le mieux possible ${ }^{22}$.

23

18 Le 14 janvier 1848 , le comte remporte à la tribune un de ses beaux succès ${ }^{24}$. Le discours sur la Suisse s'élabore au fil du mois de décembre 1847, dans un échange épistolaire constant avec Foisset. «Je vous demande de jeter sur le papier toutes ces impressions sur Lucerne. Adressez-les moi comme une simple lettre : je les jugerai avec impartialité et, s'il y a lieu, j'en ferai l'objet d'une communication au Correspondant » ${ }^{25}$. En fait, Foisset, en une dizaine de lettres, amènera Montalembert vers la tribune en faisant de son intervention, non pas une défense des libertés religieuses strico sensu (ce qui permet d'esquiver le problème des congrégations et des jésuites), mais un discours fondamentalement politique : « c'est un discours politique qu'il faut faire, pas un discours catholique $"{ }^{26}$. Ce jeu de passe-passe repose sur un inversement de perspective : il ne s'agit plus de montrer la Suisse catholique oppressée mais de l'exposer au travers de la Suisse «radicale» oppressive. En ligne de mire: passer d'une question de liberté religieuse nationale à une question d'ordre social et politique de dimension européenne.

de la préparation de ses grands discours, Montalembert entreprend une analyse documentaire souvent poussée du sujet qu'il a à traiter ${ }^{27}$. Quelles sont les informations dont il dispose pour son discours sur la Suisse?

Dès 1845, l'année de la formation du Sonderbund, Montalembert est en contact avec Constantin Siegwart-Müller, leader de l'alliance des sept cantons, qui a pris contact avec lui parce qu'il est le chef du mouvement catholique et, surtout, parce qu'il est homme politique, à même d'intervenir auprès des hommes du gouvernement. Siegwart-Müller lui demande une double intercession.

21 D'une part, il s'agit d'éclaircir la position du gouvernement français, qui s'était engagé (via l'ambassadeur en Suisse, le comte de Pontois) à livrer des armes, que Siegwart-Müller s'est vu finalement refusées ${ }^{28}$. À Strasbourg, où il venait chercher « 2000 fusils, quatre canons, six obusiers...", on lui apprend que le ministère n'accorde finalement pas le crédit envisagé. Siegwart-Müller demande à Montalembert de « court-circuiter » Guizot en s'adressant directement à Louis-Philippe.

D'autre part, Siegwart-Müller demande au comte de lui proposer des chefs militaires aptes à prendre la direction des opérations. Montalembert se tourne vers son réseau polonais, ces émigrés dont il a défendu la cause tout au long de l'année. De Skrynecki ${ }^{29}$ à Bruxelles, il reçoit des conseils stratégiques pour la défense de Lucerne (organisation de 
combats de rues) qu'il transmet à Siegward-Müller. Finalement, celui-ci repoussera les officiers mercenaires polonais proposés, essentiellement parce que ceux-ci réclament des sommes trop élevées ${ }^{30}$. Ainsi, Montalembert est informé depuis 1845 de la situation de la Suisse et se tient au courant de son évolution par toute une série de connexions : réseau lié au Sonderbund (E. Schérer) ou plus spécifiquement ecclésiastique (tel Dunoyer, curé de Genève, ou dom Brande, dominicain de l'abbaye d'Einsielden) ou encore plus " officiel » (De Grénus, consul de Belgique, sans doute lié à ce qu'on pourrait appeler le « réseau Mérode ${ }^{31}$ ).

Foisset tient une place de choix dans ce réseau. Son gendre, le Dr Édouard Dufresne, catholique de Genève, est impliqué dans l'affrontement qui se dessine ${ }^{32}$. En de nombreuses lettres il tient son beau-père au courant de l'évolution de la situation. Ces lettres, Foisset les résume à Montalembert et les fait publier dans le Correspondant et le Spectateur. Elles servent à alimenter des chroniques qui deviennent régulières à partir de novembre 1847. Foisset fait même office de passeur en portant à Genève les lettres que le comte envoie à Müller.

Essayons de sonder le discours du 14 janvier selon ses buts, sa tactique avant d'en exposer les ressorts idéologiques et culturels.

Le but du discours projeté à la Chambre des Pairs est de faire de la question suisse une question de droit et d'ordre en soulignant que le prétexte religieux est le premier pas dans une offensive de plus large envergure et que, précisément, le choix de ce prétexte est un signal fort. En somme, escamoter le problème religieux pour mieux le rendre évident et crucial. Il s'agit d'abord de dissiper l'assimilation réalisée par le "giobertisme » entre le Sonderbund et la cause autrichienne. Les sept cantons réclament seulement la liberté qui leur est due et que garantit le Pacte fédéral de 1815. Le catholicisme est dans cette affaire du côté de la liberté et dans le camp du droit tandis que le radicalisme, majoritaire à la Diète, est à l'inverse synonyme d'oppression et d'illégalité reposant sur la force. Au final, il convient donc de montrer, comme le dit Foisset, que le radicalisme n'est pas la démocratie, mais le «despotisme sous sa forme populaire " ${ }^{33}$. De là découle une volonté de sensibiliser le gouvernement aux devoirs de la France. Son droit de regard sur la question suisse s'impose parce que ses intérêts sont en jeu. D'où, trop " tendrement " selon Foisset, une charge contre l'impéritie de Guizot depuis trois ans, ce qui, notons-le, paraît passablement injuste. L'essentiel du discours réside dans cette mise en garde contre l'attentisme de la France, qui est une abdication devant la diplomatie anglaise, et une erreur d'estimation de la nature des événements. Ici, il éclatera que la question suisse est de dimension nationale et européenne.

La tactique vise à désarmer les préventions contre l'orateur catholique. Montalembert se placera pour cela dans le prolongement de l'attitude prise à l'égard de la Pologne et usera de son crédit passé pour montrer sa constance politique. Le fait d'avoir condamné la remise en cause de l'ordre européen par les puissances conservatrices lui donne autorité pour dénoncer le péril radical : d'où le "vous direz: le radicalisme cracovise la Suisse». La tyrannie n'a pas de parti : «Il ne faut pas plus flatter les peuples que les rois », dit-il à la tribune. En somme, se placer sur le terrain des libertés avant de se placer sur celui des libertés religieuses. On sait pourtant que c'est bien parce que la cause fut catholique qu'elle provoqua tant d'émoi chez nos deux épistoliers. Le discours se résume en fait à un portrait-charge $\mathrm{du}$ radicalisme. L'orateur appuie son discours sur des exemples d'iniquités et de violences puisés dans sa correspondance (et dans celle de FoissetDufresne) et dans la presse. Il remonte l'histoire du XIX ${ }^{\mathrm{e}}$ siècle pour montrer que 
l'histoire de la Suisse depuis 1815 se résume en un travail patient d'éradication des forces d'opposition par "les menées d'une minorité ", selon l'expression de Foisset qui reconstruit longuement pour le comte cette histoire ${ }^{34}$. Dans cette éradication sont habilement mêlés protestantisme et catholicisme ${ }^{35}$ : déconfessionnalisation des enjeux et concession de bon aloi à Guizot. Dans la correspondance entre les deux hommes, cette association n'est pas faite : le radicalisme suisse se confond avec le protestantisme, ce qui fausse considérablement l'appréhension des événements ${ }^{36}$. Enfin, et c'est le cœur du discours, la sombre description des exactions des radicaux suisses sert à faire prendre conscience, et crainte, de ce qui se passe en France. L'orateur le résume brillamment: " on ne s'est pas battu en Suisse ni pour ni contre les jésuites, ni pour ni contre la souveraineté cantonale; on s'est battu contre vous et pour vous». Et plus loin : «je ne viens pas parler pour des vaincus, mais à des vaincus " ${ }^{37}$. Le déplacement de la mise en perspective est net et incisif.

Foisset avait insisté sur la nécessité d'amener le débat sur "l'étroite connexité » des événements suisses et des banquets français. Plus encore, une généalogie à sa manière, ou pour mieux dire un télescopage, lui permettait de mettre à jour un procès historique parallèle : le présent suisse dévoilait le sens du passé proche français. Du même coup, il chatouillait les hantises de la Monarchie de Juillet. Voici ce qu'il spécifiait dans un de ces articles : « La prise de Fribourg est un événement français. Qu'on ne s'abuse point, c'est le drapeau vaincu à Lyon, en 1831 et 1834, qui se relève de l'autre côté du Jura, comme une menace permanente désormais contre la plus vulnérable de nos frontières ${ }^{38}$. Cette citation est reprise par l'orateur : «Le drapeau que vous avez vaincu à Lyon, en 1831 et 1834 , ce drapeau-là est aujourd'hui relevé de l'autre côté du Jura (Sensation) » ${ }^{39}$. Au fil du discours, l'apport du magistrat bourguignon est patent. Ici, c'est l'assimilation des hommes de la Diète à ceux des banquets (Foisset avait fait un article sur le discours de Ledru-Rollin à Chalon ${ }^{40}$ ). Là, c'est la responsabilité de certains écrivains français, allusion claire à Lamartine (« cette plume qui a poétisé ce qu'il y avait de plus triste dans notre révolution " ${ }^{41}$ ) dont l'Histoire des Girondins vient d'être "exécutée " par Foisset dans le Correspondant où il accuse son compatriote de spéculer sur les mauvaises passions du temps. Ainsi, ce travail à quatre mains montre, les jésuites exceptés, la symbiose entre les deux amis : « Oncques nos cœurs ne vibrèrent plus à l'unisson » ${ }^{42}$.

Foisset comme Montalembert perçoivent Genève et Berne comme le centre d'une internationale radicale, tissant patiemment sa toile, sorte de réplique du mythe jésuite, et c'est pourquoi l'orateur rappelle que ce lieu cosmopolite, fut et reste le refuge de tous les réprouvés d'Europe. Tel l'infatigable Mazzini, qui, avec le général Ramorino, lance en 1834 une opération, pour le moins aventureuse, visant à soulever la Savoie et secouer le régime piémontais : le péril international ne manque donc pas de signes avant-coureurs. $\mathrm{Si}$, de nos jours, les historiens dissocient les événements suisses du mouvement des nationalités de 1848, il est clair que cet avenir proche confortera la filiation opérée " préventivement » par nos deux hommes. Cette approche doit contribuer à expliquer les positions de Montalembert lors des années 1848-1852.

Comprendre les ressorts de la construction de ce discours, c'est-à-dire de l'analyse des événements, c'est toucher aux liens ambigus entre politique et religion dans les positions du mouvement catholique. Ici, la critique que Foisset porte à l'encontre de l'ouvrage de Lamartine éclaire les fondements et les motivations de l'attitude à l'égard de la question suisse : « On se persuade que rien n'a ressemblé à 1793 dans le passé, que rien ne saurait y ressembler dans le présent, rien dans l'avenir; que la Terreur n'a été qu'une de ces 
monstruosités qui n'arrivent qu'une fois en soixante siècles; que les acteurs de ce mélodrame sanglant étaient des êtres d'exception comme on n'en reverra jamais. Rien n'est moins juste de ce point de vue [suit la liste des activités des futurs révolutionnaires]. Aucun d'eux peut-être, en 1789, ne se serait cru lui-même capable de la moitié de ce qu'ils devaient faire. Mais l'ouragan vint: le vertige des révolutions et celui de la peur bouleversèrent à l'envi toutes ces têtes au-delà de toute prévision humaine. Que celui-là entende qui a des oreilles pour entendre !» ${ }^{43}$

C'est toute une approche de la dynamique révolutionnaire qui est ici exposée, sur les fondements d'une culture religieuse (l'infirmité humaine), et une approche politique qui est formulée (l'impuissance de la volonté humaine à transformer la réalité). Ce texte, rédigé avant que la crise n'éclate, alors que la campagne des banquets débute en France, trahit une perception du présent que l'écrasement du Sonderbund, puis bientôt les événements romains, ne peuvent que confirmer. Il est question de surdité et d'aveuglement. L'imprévoyance remet l'avenir aux mains du hasard, c'est-à-dire de l'aventure, donc du pire. Cette mise en garde est de même nature que celle que Montalembert adresse du haut de la tribune: l'avenir avance masqué et c'est à la clairvoyance de l'homme politique de déceler dans le présent les secousses de l'ébranlement. Or, les signes de cet ébranlement, c'est dans les attaques contre la religion et les libertés religieuses qu'ils se révèlent. Assurément, une période, le XVIII ${ }^{\mathrm{e}}$ siècle, et un homme, Louis XVI, incarnent, au mieux, cette insouciance, au pire, cette incurie. Ainsi, l'avenir proche est gros du passé : c'est au travers de ce prisme déformant que le présent est interprété et, par conséquent, défiguré. C'est ce qui explique que l'action de la majorité de la Diète est comme "naturellement saisie» par nos deux observateurs: comme par réflexe, ils imaginent sans mal ce que la propagande du Sonderbund diffuse. Il ne fait nul doute que la religion est le dernier rempart avant l'invasion du radicalisme.

31 Cette approche est conditionnée par une culture historique : une culture imprégnée des auteurs de la contre-révolution qui fausse l'interprétation des événements. Ceux-ci ne sont plus interprétés pour ce qu'ils sont, mais comme des signes de retour de ce qui fut. La Suisse radicale est assimilée à la Suisse protestante. L'action de la Diète est assimilée à celle de la Terreur jacobine ( «on a dit qu'il y avait là toute notre révolution sans l'échafaud, cela n'est que trop vrai » ${ }^{44}$ ) et dans le prolongement logique de l'esprit de la Réforme, selon la filiation établie par les penseurs de la contre-révolution. Le Sonderbund, c'est la Vendée. En France, Lamartine, Michelet, ou Blanc constituent des signes annonciateurs : la réhabilitation préside au passage à l'acte «il est trop tard; le club des jacobins est déjà rouvert, non pas en fait et dans la rue, mais dans les esprits » ${ }^{45}$. Quant à la campagne des banquets ou aux méthodes des radicaux en Suisse, y résonne l'écho des tribuns du sans-culottisme: "Un homme monte sur une table au milieu de 3 à 400 compères : il se propose pour chef d'un gouvernement provisoire - acclamations - il désigne ses collègues - acclamations - il proclame un nouveau mode d'élection - acclamations. Quelle comédie! ${ }^{46}$ Cette hantise du spectre de la Révolution ramène résolument et ouvertement la défense des intérêts religieux vers la politique conservatrice.

La défaite du Sonderbund, pour ainsi dire sans combattre, provoque une profonde désillusion et une réflexion pessimiste sur le monde contemporain où les valeurs anciennes, viriles disent parfois nos auteurs, s'étiolent : « Le peuple de la Suisse primitive est catholique : mais c'est le catholicisme du Moyen Âge moins l'énergie » ${ }^{47}$. Foisset, dans un article du Spectateur, avait décrit la mobilisation des sept cantons, toute populaire, 
nationale, volontaire, religieuse. En somme, la prise d'armes de la Vendée, déjà mythifiée. "L'abâtardissement » des mœurs contemporaines est un leitmotiv de la correspondance d'hommes qui veulent agir dans un siècle qu'ils n'aiment et n'estiment que fort peu : leur volontarisme politique s'échoue sur la nostalgie du Moyen Âge et du XVII ${ }^{e}$ siècle. Ainsi, Montalembert, dresse le même constat pessimiste mais qui débouche sur sa lutte fondamentale et rend à son attitude sa cohérence : «Je ne croyais guère à une victoire complète du bien : mais je croyais à une lutte qui eut excité l'admiration et la compassion de l'univers. Cette lutte valait une victoire ; car c'est ainsi que la Vendée a vaincu, et nous a conservé l'Église en France. Dieu en a jugé autrement : nous buvons le calice jusqu'à la lie : et quelle lie! nous n'avons pas même le droit de blâmer nos frères suisses de leur lâcheté, car nous ne ferions pas même ce qu'ils ont fait. La Vendée elle-même de nos jours, ne supporterait pas un mois de blocus, ni deux jours de combats. [...] Je crois vous avoir dit que selon moi la principale [cause de notre abaissement] était la pitoyable éducation religieuse des chrétiens d'aujourd'hui » ${ }^{48}$.

Le discours fut généralement bien accueilli : les 136 lettres de félicitations conservées dans les archives l'attestent. Notons-le : l'écrasante majorité de ces lettres proviennent de membres de l'aristocratie. L'épiscopat y est très discret ${ }^{49}$. Signe du repositionnement, Mgr Clausel de Montals, l'évêque de Chartres souvent en délicatesse avec le mouvement catholique, félicite chaleureusement Montalembert. Plus critique, Mgr Parisis, compagnon de lutte du mouvement catholique ${ }^{50}$ : «Désavouer les sympathies des hommes d'État pour notre amour de l'ordre et du droit, ce ne serait ni juste ni prudent ; les accueillir sans réserve, c'est laisser entrevoir que nous entendons l'ordre et le droit comme eux, ce qui n'est pas, car ils ne les entendent que du côté apparent et matériel [...]. Il ne faut certainement pas sacrifier le présent pour des calculs aventurés sur l'avenir; mais il faut aussi prendre garde de se laisser tellement envahir par les préoccupations même légitimes des dangers actuels que l'on se jette dans des alliances qui puissent devenir plus tard des chaînes oppressives pour la liberté religieuse ${ }^{51}$.

Il n'est pas indifférent de noter que l'évêque de Langres adopte finalement les positions que Montalembert et Foisset défendaient quelques temps auparavant. Comme lui, ils pensaient qu'il fallait réserver l'avenir, détacher le mouvement catholique des assimilations partisanes. Cette assimilation, dont Foisset et Montalembert s'étaient toujours défendus, ils l'ont réintroduite, sous l'effet de leur hantise, en faisant finalement cause commune avec un régime parlementaire conservateur. Ce régime qui va bientôt va tomber à la grande surprise et au grand désarroi des deux hommes... sous les coups d'une révolution dont ils agitaient le spectre et qui les surprendra finalement presque incrédules.

pronunciamento ${ }^{52}$

Le discours prononcé le 11 janvier 1848 sur l'Italie ne semble pas avoir frappé les esprits. Dans le Correspondant, Charles Lenormant, rédacteur en chef de cet organe de la nébuleuse catholique libérale, reconnaît qu'il a été éclipsé par celui sur la Suisse. L'inversion chronologique choisie ici est en fait un rétablissement : l'élaboration du discours sur la Suisse précède celle du discours sur l'Italie, même si les compositions en tant que telles sont simultanées (début janvier 1848). C'est un point de vue stratégique qui présida à l'ordre des interventions : il semblait, tant à Foisset, qui en fait la mention expresse, qu'à Montalembert, qu'il était préférable de commencer par l'intervention la plus épineuse tandis qu'il serait facile de rallier l'opinion conservatrice sur la question suisse. Plus encore que pour le Sonderbund, Montalembert a longtemps hésité à prendre la parole sur 
les événements romains. Le comte le confesse dans ses carnets en janvier 1848 : c'est le magistrat bourguignon qui vient d'emporter sa décision. Finalement, les thèmes que l'orateur voulait aborder à propos de la question des jésuites en sont venus à se décliner en deux discours où ceux-là sont éclipsés. Montalembert a reçu des sollicitations diverses pour intervenir sur l'Italie : au premier rang de celles-ci, il faut citer le cardinal Cadolini (archevêque de Ferrare, ville que l'Autriche vient, en forme d'intimidation, d'occuper en juillet 1847 et qui cristallise les sentiments nationaux) et le comte Balbo, favorable à l'établissement de l'unité sous l'égide de la dynastie piémontaise. Ces deux hommes constituent même l'impulsion première. C'est à la lecture de ces deux lettres que Foisset s'emporte : «Je suis stupéfait que vous ne vouliez rien dire de Rome ${ }^{53}$.

En juin 1846, nos deux hommes suivent évidemment avec assiduité le conclave. Il faut bien avoir présent à l'esprit que depuis 1846, Montalembert suit plus que jamais ce qui se passe à Rome. Il craint un désaveu de la campagne relative à la liberté d'enseignement qui secoue la France depuis 1843 : en ce qui concerne l'action politique au nom des intérêts de la religion ou l'action par la presse. Il a même pris soin d'écrire à Grégoire XVI, puis à Pie IX, afin de justifier l'action du mouvement catholique et de l'épiscopat français. Il serait prématuré, dans l'état des recherches, de dresser ici les réseaux d'informateurs en Italie. Une chose est certaine : il est varié et riche, à la fois ecclésiastique, mondain et politique. Au demeurant, pour suivre les deux hommes, il suffit de suivre L'Ami de la religion ou l'Univers, qui restent leurs sources privilégiées d'informations et que, de surcroît, ils alimentent.

L'élection de Pie IX ne soulève pas d'emblée l'enthousiasme du seul fait qu'il n'est pas connu de nos deux épistoliers. L'amnistie des prisonniers politiques est favorablement saluée parce qu'elle constitue une rupture symbolique avec l'influence autrichienne. L'adhésion est plus nette à la suite à l'entrevue que l'abbé Dupanloup obtient de Pie IX. L'abbé décrit longuement l'audience et affirme que le Pape est pleinement favorable à l'action du mouvement catholique en faveur de la liberté d'enseignement. Il rapporte cette parole du Pape, dont le comte s'enorgueillit: «M. de Montalembert, son nom seul est un éloge ${ }^{54}$.

Toutefois, en juillet-août 1847, l'inquiétude paraît s'installer. Le mois de juillet a vu se dérouler à Rome des troubles importants, fondés sur les rumeurs d'une répression du mouvement en cours. Sur les murs de la ville, des affiches apparaissent où les noms des coupables supposés sont mentionnés. Le P. Ventura, s'appuyant sur la notoriété que lui a valu son éloge d'o'Connell, vient à bout d'une de ces fermentations ${ }^{55}$. C'est sans doute à cet événement que Foisset fait allusion lorsqu'il déclare : «Le P. Ventura m’a fait plaisir. Mais je le crois capable de s'aventurer. Je n'aime pas qu'il ait à descendre de son couvent pour dissoudre un commencement d'émeute ${ }^{56}$. Cette suspicion, touche bientôt la personne de Pie IX : «Quand à Pie IX, je soupçonne qu'il peut être inférieur à sa tâche ». $\mathrm{Au}$ début de 1848, le jugement est encore plus dubitatif : « Il est certainement le meilleur des hommes et le meilleur des prêtres. Mais, à entendre le comte Balbo, il n'a pas de plan ; il donne au jour le jour ce que l'opinion lui demande. Il plie devant les journaux, laisse la censure défaire ce qu'il fait, approuver ce qu'il improuve. Il ne tire aucun parti de sa popularité personnelle pour faire reculer l'ennemi ; aucun parti des sentiments religieux du peuple romain contre Mazzini et consorts. En somme on ne le sent pas de force à résister au torrent et on se laisse entraîner on ne sait où (assez doucement, il est vrai, jusqu'ici du moins). On règne, on ne gouverne pas [...]. Quoiqu'il en soit, j'aime mieux Louis XVI sur le trône de Rome, que le cardinal Lambruschini [chef de file des zelanti]. Au 
point de vue de l'avenir, il n'y a pas de comparaison ${ }^{57}$. C'est dans ce contexte que le magistrat pousse l'orateur à la tribune.

Il est clair que pour les deux hommes Pie IX ne contrôle plus les événements. Il ne réforme plus mais concède. L'agitation de la rue rejoue encore une fois le processus de la Révolution. Une minorité profite de l'action du Pape, cherche à le pousser au-delà de ses volontés et manipule les foules. Pie IX est débordé par cette aile. Dans la réponse prévue pour l'évêque de Ferrare, en novembre, Montalembert envisage les difficultés à venir du Pape comme inéluctables. Il propose, très révérencieusement, deux lignes directrices au cardinal. La première est d'amener les catholiques à faire bloc autour du souverain pontife afin de le trouver fort au moment des difficultés. C'est notamment pour cela qu'il condamne sévèrement l'action de Gioberti qui sème le trouble chez les catholiques et risque de diviser les forces: "Si nous marchons au schisme (et la chose n'est pas impossible), Gioberti en sera l'antipape $"{ }^{58}$. Deuxième ligne directrice, complément naturel de la première, il faut user des moyens qu'offre la liberté de la vie politique (association, presse, publicité, débat politique) pour mobiliser les masses catholiques. Ici, les catholiques italiens doivent faire un apprentissage accéléré, indispensable pour assurer leur crédibilité dans la lutte : « l'avenir ne tardera pas à mettre leur dévouement à l'épreuve : il ne faut pas que d'ici là ce dévouement soit suspect ${ }^{59}$. Sans le dire, il est évident que Montalembert propose l'exemple du mouvement catholique en France. C'est au nom de cette logique que Foisset poussera le comte à la tribune : d'une part, montrer l'exemple d'une liberté catholique bien entendue, d'autre part, faire entendre une voix qui sera comme un message de ralliement, un appel à la prise de conscience.

Le tournant véritable du point de vue de nos épistoliers paraît se situer en décembre, c'est-à-dire après les manifestations du trois de ce même mois célébrant la victoire des armées de la Diète en Suisse. L'Autriche et les jésuites y sont conspués. La presse échappe à la censure. Une brochure publiée pour exposer la "vraie » nature du Sonderbund est brûlée. L'opinion publique est dans la rue, dans les cafés. Inutile d'insister une fois encore sur ce que ces scènes évoquent : la terreur jacobine est en gestation. Il est plus intéressant en revanche de noter le lien qui s'établit entre la question suisse et les événements romains. La défaite du Sonderbund constitue un tournant dans l'appréciation de nos deux épistoliers. Ils critiquent sévèrement la timidité du soutien de Pie IX à la cause des catholiques suisses. Ce qui se passe à Rome atteste et confirme, à leurs yeux, un péril européen. En ce sens, il ne fait nul doute que les deux interventions de janvier sont le diptyque d'un même discours politique: la religion est le dernier rempart face à l'invasion du radicalisme. Le P. Ravignan, qui se trouve à Rome, estime devoir à Montalembert «toute la vérité pour [le] mettre à même de bien apprécier la situation »: "Jugez l'Italie par la France et la Suisse radicales : vous ne vous tromperez pas » ${ }^{60}$.

41 Pourtant, le ton du discours du 11 janvier se voudra moins catastrophiste parce qu'il ne s'agit pas, comme avec la Suisse, de constater pour fustiger, mais cette fois de prévenir pour engager : c'est pourquoi le discours sur l'Italie vient après la "démonstration " suisse. Montalembert reprend, à peu de chose près, le plan de discours que lui dresse Foisset. En guise d'introduction, il souligne l'universalité de la popularité de Pie IX et l'étrange silence de la France à cet égard : c'est ce qui permet au comte d'intervenir lors du projet d'Adresse (paragraphe 6) où la Chambre s'associe au gouvernement pour l'encouragement aux «progrès que chaque pays pourra accomplir dans action propre ». C'est sur ce point que Foisset pousse l'orateur: il faudra, dans un premier temps, s'étonner candidement qu'il ne soit pas fait nommément mention de l'action de Pie IX. La 
suite du projet d'Adresse stipule que ces progrès devront se réaliser « de concert entre les gouvernements et les peuples»: ce qui permet d'enchaîner la protestation à l'étonnement premier, en soulignant que Pie IX ne doit pas être " court-circuité » dans ses relations avec son peuple par la minorité susmentionnée. Les radicaux sont décrits, occasion qui permet à l'orateur une ironie habile : « une minorité dont on peut dire bien justement, comme de celle qui s'est rencontrée ailleurs, qu'elle n'a rien appris et rien oublié » ${ }^{61}$. C'est aussi se placer en homme modéré, en homme partisan du « juste milieu ». Il appartient donc à la France de manifester son intérêt pour les affaires romaines et pour l'action de Pie IX. La France a un rôle à y jouer et une opportunité politique à saisir puisque l'Autriche n'y peut jouer aucun rôle positif. L'orateur prend soin de distinguer les radicaux, infime minorité, des "impatients » et des « utopistes ", dont Montalembert fit partie dit Foisset, probable allusion au début des années 1830. Cette distinction exprime l'indulgence de mise à l'égard de l'inexpérience italienne en matière de libertés. « Il faut l'avertir affectueusement, fraternellement », dit l'orateur. Attitude condescendante. Sans doute. Mais plus encore stratégique. Après la lecture de la lettre de Balbo, Foisset écrivait à Montalembert que « les illusions méritaient d'être ménagées avec le plus grand tact » et qu'il était « bon de mêler à notre protestation un grain d'encens pour le peuple romain [... ], qu'on ne saurait, pour se faire écouter au-delà des monts, parler du mouvement italien avec trop de sympathie, et des écarts avec trop d'indulgence ${ }^{62}$. Cette sollicitude, Lenormant la résume moins diplomatiquement dans son compte rendu du discours quand il dit qu'il faut laisser ces paroles parcourir leur chemin : « qu'elles retentissent au milieu des scènes humiliantes et puériles auxquelles donnent lieu l'inexpérience du patriotisme italien ${ }^{63}$.

Le discours de Montalembert est donc une manière de mise en garde. Mais il n'en a pas le ton. C'est que d'autres objectifs se font jour. Il s'agit, une fois encore, de ne pas donner prise à l'ennemi. Il convient donc de répondre aux accusations : le clergé français suit le souverain pontife comme un seul homme et acquiesce aux réformes ${ }^{64}$ tout comme il s'est rallié dorénavant au régime en place. Le Pape incarne les idées que défend le mouvement catholique depuis 17 ans, dit le comte. C'est-à-dire qu'il adhère au parlementarisme « moderne » et s'identifie avec le parti de la résistance : « la mission actuelle du Pape n'est autre que la mission de la France de 1830 » et il faut que les Romains «constituent un grand parti politique, comme celui qui a sauvé la France après 1830 [qui] aura pour base ou pour auxiliaire le catholicisme personnifié dans son chef ${ }^{65}$. C'est ce que Pie IX est en voie de réaliser. Pour mieux convaincre l'auditoire, le comte suit les conseils de Foisset en faisant appel à une longue citation de ce qu'ils nomment "l'adresse de New York»: l'adhésion des catholiques américains à l'œuvre de Pie IX offre l'avantage de venir d'outre-Atlantique, cette démocratie sage et religieuse mise en lumière par Tocqueville. Montalembert comme Foisset connaissent trop bien les réticences du clergé français, mais les événements offrent une tribune à leurs opinions. L'action de Pie IX, compliquée presque heureusement par sa situation, apparaît alors être l'opportunité tant attendue : convertir le clergé et les catholiques aux « libertés modernes » et à l'action unitaire, une occasion, en quelque sorte, de leur forcer la main sous l'emprise de la nécessité. C'est ce que Foisset appelle son propre pronunciamento réalisé dans les colonnes du Spectateur: «Or, par un déplorable mal-entendu, un antagonisme réputé implacable s'était établi entre les représentants de ces idées et les représentants naturels de la foi catholique. Je n'ai pas le temps de dire aujourd'hui à quoi a tenu ce mal-entendu. Qu'il me suffise de rappeler qu'en 1787, l'assemblée du Clergé de France avait demandé les États Généraux ; 
que les cahiers du Clergé, rédigés en vue de cette grande manifestation nationale, étaient pleins de vues de réformes; que le clergé se réunit promptement au Tiers-État en juin 1789 ; que le divorce ne s'opéra point par la confiscation des biens d'Église, mais par la Constitution civile du clergé, constitution qui faisait nommer les évêques par ceux qui n'allaient jamais à la messe; que ces mêmes prêtres qui refusaient le serment à cette constitution, à cette oppression des consciences, prêtèrent avec empressement le serment à la liberté et à l'égalité, décrété par l'Assemblée législative (1792); qu'ils prêtèrent serment aussi à la Révolution faite homme dans la personne de Napoléon, et qu'ils ne se séparèrent de lui que lorsqu'il se fit le persécuteur de l'Église en la personne de Pie VII ; qu'enfin le clergé contemporain n'a pas fait la moindre opposition à la révolution de Juillet, et qu'il n'est intervenu depuis 17 ans que dans une seule question politique, celle de l'enseignement, et c'est une question de liberté $"{ }^{66}$.

Aussi, le discours est l'occasion de prendre d'assaut les préventions et d'impliquer le clergé de France. Pour cela, il convient de décrire l'action mesurée et éclairée du souverain pontife. La distinction entre radicaux, impatients et utopistes tendait déjà à limiter l'influence des premiers, donc l'instabilité réelle. Dans le Spectateur, Foisset est clair: "Tout [ce que le Pape] a fait, il l'a fait spontanément; il ne s'est rien laissé demander [...]. Il est demeuré maître en droit et en fait » ${ }^{67}$. L'orateur souligne : « Non le pape n'a pas mal fait; non il n'a pas été imprudent, il ne doit regretter rien de ce qu'il a fait ${ }^{6}{ }^{8}$. Évidemment, cette position est en contradiction avec le sentiment pour le moins inquiet de nos deux épistoliers dans cette période. On comprendra aisément qu'ils ne peuvent publiquement qu'afficher une confiance sereine. Mais le discours de Montalembert, tout politique qu'il se voulût, fut trop religieux par son fond pour ne pas entretenir la confusion. Certes, il fallait sensibiliser le gouvernement aux événements romains et faire en sorte que l'Italie entende la voix de la France. Bien entendu, il fallait défendre l'autorité du chef de l'Église. Mais, finalement, voici ce " clergé contemporain, qui n'est pas intervenu depuis 17 ans que dans une seule question politique ", voici le mouvement catholique, voici le Pape lui-même, associés à une forme de régime politique : celui de la Monarchie de Juillet. On sait que cette confusion est pour le moins discutable. Nos épistoliers le savaient aussi. Mais les besoins de la cause méritaient bien une petite entorse à la réalité. Mais il leur avait semblé que, pour des raisons stratégiques propres à la défense des intérêts religieux, il fallait s'associer à ce régime. C'était risquer l'assimilation hier encore contraire à tous leurs vœux et, finalement, confirmer les accusations de duplicité portées à l'égard du mouvement catholique. Décidément, il n'y eut pas de parti catholique au-dessus des forces politiques.

Les conséquences de ces deux discours sont importantes puisqu'ils signent la fin du mouvement catholique. On place la scission traditionnellement en 1848-1849 et Montalembert l'imputera à Louis Veuillot. En fait, la réception des discours de janvier marque la vraie rupture. Avec Ozanam, qui reprendra bientôt le terme de «barbares " réservé par Montalembert aux radicaux dans son discours sur la Suisse, mais sous la plume de l'historien, ce sera cette fois " pour y passer » ${ }^{69}$. Avec Lacordaire, qui écrit à son bientôt "cher ancien ami", une dernière lettre, encore d'amitié et, somme toute, de fidélité. Les deux discours, surtout celui du 14 janvier, achèvent pour lui un éloignement qui avait débuté avec... la question des jésuites en 1844 : 
«Je voyais depuis 1843 un intérêt secondaire dans l'Église prendre le pas sur l'intérêt universel, et je comprenais que cet intérêt menacé à Rome et dans toute l'Italie faisait des efforts inouïs pour se substituer plus que jamais à tout le reste [...]. Je n'ai pas l'espérance de t'éclairer. Tu me parais depuis longtemps sous des influences auxquelles ton esprit s'est habitué, et où tu as cru trouver pour le bien de sérieux appuis. Le temps seul renversera tes convictions présentes, et te fera entendre que tu eusses été plus fort avec moins d'alliances, parce que tu eusses mieux gardé la véritable perspective de l'avenir et de la vérité [...]. Il est bien dur, après 17 ans, de me trouver en désaccord avec toi sur des points aussi capitaux. [...] Mais aussi tu ne seras pas étonné de me voir suivre avec vigueur la route où je suis entré, et où il est trop possible qu'un jour nous arrivions jusqu'à des dissentiments publics [...].

47 J'ai préféré t'écrire parce qu'une lettre reste : et que d'ailleurs il m'eût été trop pénible de t'exprimer de vive voix ce que l'amitié et l'honneur me commandaient de te faire savoir » 70 .

Les bases des divorces de 1848 sont posées, tandis que, simultanément, un rapprochement avec Veuillot, certes temporaire, se profile. À la suite du discours sur la Suisse, celui-ci écrivait à $\mathrm{Du} \mathrm{Lac}$ : «Vous ne pouvez vous faire une idée du succès de Montalembert [...]. Le voilà, sans avoir rien perdu de son caractère catholique, à la tête des idées conservatrices ».

\section{NOTES}

1.. Archives départementales de la Côte-d'Or (Arch. dép. Côte-d'Or), archives Montalembert (Arch. Montalembert), dossier 26, Journal manuscrit, 1847. La présente contribution participe d'un travail en cours sur les archives du comte de Montalembert et celles de Théophile Foisset déposées aux archives départementales de la Côte-d'Or.

2.. Ibidem.

3.. Arch. Montalembert, dossier 450, Foisset à Montalembert, Sainte Geneviève 1847.

4.. Foisset, suivi en cela par les membres du Correspondant, n'aimait guère l'appellation " parti catholique ». Il aspirait à une action politique qui ne soit pas partisane. Montalembert lui-même a classé les dossiers relatifs à l'action du groupe sous la dénomination « mouvement catholique », ce qui contourne l'ambiguïté sans la dissiper. Par ailleurs, mais pour d'autres raisons, l'historien ne peut concevoir la notion de parti que comme pour le moins abusive en ce qui concerne cette période.

5.. Quatre interventions en 1847. Montalembert s'inscrit ici dans une ligne directrice ancienne (celle de l'Avenir) ; voir Nicole TAILLADE, « Montalembert, Lamennais et la Pologne ", dans Actes du colloque Lamennais (1982) - Cahiers menaisiens, $\mathrm{n}^{\circ}$ 16-17, 1983-84, pp. 59-70.

6.. Théophile Foisset est à cette époque juge d'instruction à Beaune. Il effectuera toute sa carrière en Bourgogne, qu'il refusera de quitter malgré les invitations faites par ses amis parisiens (direction des Cultes sous Falloux en 1849 ou direction d'organes de presse). Toutefois, il est en liens constants avec le mouvement catholique dont il est, de l'avis de 
tous, un membre actif et écouté. Ce que prouve l'abondante correspondance avec Montalembert (plus de 600 lettres pour chacun des épistoliers de 1837 à 1870).

7.. Joseph-Théophile FOISSET, Catholicisme et protestantisme, Dijon, F. Hémery, 1845.

8. Il est impossible d'entrer dans le détail de cette brouille qui couve depuis 1845. Disons simplement que les attaques assez peu sélectives de l'Univers compliquent la stratégie du mouvement catholique telle que l'entrevoient Montalembert et Foisset.

9.. Sur le Sonderbund, une synthèse événementielle récente : Pierre DU BOIS, La guerre du Sonderbund, Paris, Éditions Alvik, 2003, 207 p. Pour une mise en perspective problématique et la bibliographie, voir Philippe HENRY, « 1847-1848 en Suisse : une révolution? ", dans Jean-Luc MAYAUD [dir], 1848 : actes du colloque international du cent cinquantenaire, tenu à l'Assemblée nationale à Paris, les 23-25 février 1998, Paris, Éditions Créaphis, 2002, p. 449-462.

10.. Arch. Montalembert, dossier 450, Foisset à Montalembert, Saint Martin de Tours 1847.

11.. Le Primato prône la réalisation de l'unité italienne sous l'autorité du Pape. Manifeste du néo-guelfisme.

12. Vicenzo GIOBERTI, Il Gesuita moderno, dans Opera, Lausanne, S. Bonamici \& Cie, 1845-1847, tomes 8 à $12,1846-1847$.

13.. Membre de la Compagnie, proche du mouvement catholique, ami de Lacordaire, dont il prend la succession comme prédicateur des carêmes de Notre-Dame de Paris de 1836 à 1846.

14.. Arch. Montalembert, dossier 26, Carnets, 20 septembre 1847.

15.. Michel LEROY, Le mythe jésuite. De Béranger à Michelet, Paris, Presses universitaires de France, 1992, 468 p.

16.. Arch. Montalembert, dossier 450, Foisset à Montalembert, Saint Martin de Tours 1847.

17.. Arch. Montalembert, dossier 450, Foisset à Montalembert, Saint Martin Tours 1847.

18.. Lacordaire à Foisset, 28 octobre 1847, dans Joseph CREPON, Lettres du P. Lacordaire à Théophile Foisset, Paris, Poussielgue, 1886.

19.. Arch. Montalembert, dossier 467, Ravignan à Montalembert, octobre 47.

20.. Charles Forbes MONTALEMBERT, Discours, Paris, Lecoffre, 1860, tome 2, p. 676.

21.. Montalembert qualifie de " trahison » et de " bassesse » l'attitude de Grégoire XVI et de Lambruschini en 1845 qui paraissent sacrifier les jésuites aux instances du ministre plénipotentiaire Rossi, dépêché à Rome par Guizot. Il considère cette défaite à l'égal du lâchage de la Pologne.

22.. Arch. Montalembert, dossier 450, Foisset à Montalembert, Saint François-Xavier 1847. 23.. Idem, 12 décembre 1847.

24.. Il ne fait évidemment pas l'unanimité, mais atteint son but en obtenant l'assentiment de la fraction conservatrice (le compte rendu favorable du Journal des Débats étant emblématique de ce point de vue).

25.. Arch. dép. Côte-d'Or, Fonds Foisset, $34 \mathrm{~J} 83$, Montalembert à Foisset, 27 septembre 1847.

26.. Arch. Montalembert, dossier 450, Foisset à Montalembert, 12 décembre 1847.

27.. Ses discours sur la question romaine ont laissé dans ses archives plusieurs cartons de notes historiques, de copies de notes de voyage, de coupures de presse et de brouillons divers.

28.. Arch. Montalembert, dossier 698-699, lettres de Müller à Montalembert, 1845-1846.

29.. Général en chef de l'armée polonaise en 1831 ; réfugié à Bruxelles. 
30.. Chlapowski, figure de la nation polonaise en 1831, acceptait de servir, contre rémunération, la cause suisse. On notera que le Sonderbund accueille dans ses rangs des représentants de l'aristocratie européenne ; voir Pierre DU BOIS, La guerre du Sonderbund, ouv. cité, p. 115. On a vu que Montalembert avait failli obéir à ce premier réflexe.

31.. Beau-père de Montalembert, un des meneurs du « parti catholique » belge.

32.. En 1847, Dufresne est la cible de la presse genevoise (Fazy) comme chef catholique. En 1848, Dufresne sera le principal rédacteur de L'Observateur de Genève, journal destiné à défendre les intérêts des catholiques genevois et financé en partie grâce à Montalembert. 33.. Arch. Montalembert, Foisset à Montalembert, 2 janvier 1848.

34.. Cette approche et les exemples donnés par Montalembert noircissent excessivement le tableau. Certes, il y eut des excès, notamment lors de la capitulation de Fribourg ou de Lucerne. Toutefois, la guerre de novembre 1847 fut aussi un guerre d'intense propagande : nos deux co-auteurs accordent trop de crédit aux informations divulguées par le Sonderbund qui exagère considérablement les exactions. On notera que dès 1846 circulent des relations de la persécution des catholiques en Suisse depuis 1815 ; voir Pierre DU BOIS, La guerre du Sonderbund, ouv. cité, p. 69.

35.. Charles Forbes MONTALEMBERT, Discours, ouv. cité, p. 681 et p. 684.

36.. On se rappelle que Foisset vient de publier un ouvrage polémique contre le protestantisme.

37.. Charles Forbes MONTALEMBERT, Discours, ouv. cité, tome 2, pp. 676-677.

38.. Le Spectateur, 20 novembre 1847 (article anonyme).

39.. Charles Forbes MONTALEMBERT, Discours, ouv. cité, p. 705.

40.. Ce banquet se déroule le 19 décembre. On voit que l'élaboration du discours se construit par et dans l'actualité.

41.. Charles Forbes MONTALEMBERT, Discours, ouv. cité, p. 691.

42.. Arch. Montalembert, Foisset à Montalembert, Sainte Catherine 1847.

43.. Théophile FOISSET, « Histoire des Girondins de Lamartine », dans Le Correspondant, tome 19,1847 , pp. 437-438.

44.. Charles Forbes MONTALEMBERT, Discours, ouv. cité, p. 687.

45.. Idem, p. 692.

46.. Ach. dep. Côte-d'Or, Fonds Foisset, $34 \mathrm{~J}$ 88, Foisset à Montalembert, « Question suisse ", discours rédigé, s.d. [décembre 1847] ; description reprise par Montalembert dans son discours, voir Charles Forbes MONTALEMBERT, Discours, ouv. cité, p. 789.

47.. Arch. Montalembert, dossier 450, Foisset à Montalembert, 16 décembre 1847.

48.. Arch. Montalembert, dossier 555, Montalembert à Dupanloup, 5 décembre 1847.

49.. Mgrs Parisis, Clausel de Montals, Affre, auxquels on peut ajouter la lettre du nonce,

Mgr Fornari. Quatre lettres de prêtres. En somme, huit membres du clergé sur 136 lettres. 50.. Il vient de publier en 1847 son Cas de conscience, sur la question de la liberté de l'enseignement, qui fait l'unanimité chez les catholiques libéraux laïques. Mgr Parisis est la figure de proue du mouvement catholique au sein de l'épiscopat.

51.. Arch. Montalembert, dossier 351, Mgr Parisis à Montalembert, 21 janvier 1848.

52.. Arch. Montalembert, dossier 450, Foisset à Montalembert, 16 décembre 1847.

53.. Idem, Foisset à Montalembert, 12 décembre 1847. Les deux lettres ont été retirées des dossiers correspondants des archives du comte. Ils font donc défaut à notre analyse. On en trouve des extraits dans R. P. Lecanuet, Montalembert, Paris, Poussielgue, 1899, tome 2, pp. 357-358.

54.. Arch. Montalembert, dossier 562, Dupanloup à Montalembert, 24 septembre et 16 octobre 1846. 
55.. On pourra ici se rapporter aux récits de l'Ami de la Religion. Le P. Ventura, religieux théatin, fut un des introducteurs du premier Lamennais en Italie. Au début du pontificat de Pie IX, il est un des conseillers du nouveau Pape. Il incline à la démocratisation des États pontificaux. Son oraison funèbre d'o'Connell (juin 1847) semble inaugurer des temps nouveaux. Ventura subira finalement une mise à l'index et se soumettra. Le religieux incarne l'héritage ambigu de mennaisianisme sur le versant italien. Il est jugé de manière très contradictoire par les catholiques libéraux de France.

56.. Arch. Montalembert, dossier 450, Foisset à Montalembert, 11 août 1847.

57.. Idem, 2 janvier 1848.

58.. Arch. Montalembert, dossier 521, Brouillon de la lettre adressée [à Mgr de Ferrare], 21 novembre 1847. Ce brouillon est corrigé de la main de Foisset.

59.. Ibidem.

60.. Arch. Montalembert, Dossier 467, Ravignan à Montalembert, 8 octobre 1847.

61.. Charles Forbes MONTALEMBERT, Discours, ouv. cité, p 667.

62.. Arch. Montalembert, ouv. cité, Foisset à Montalembert, $1^{\mathrm{er}}$ janvier 1848.

63.. Charles LENORMANT, « Des derniers discours du comte de Montalembert », dans Le Correspondant, tome 21, 1848, p. 461.

64.. La presse mettait régulièrement en doute l'adhésion du clergé aux réformes.

65.. Charles Forbes MONTALEMBERT, Discours, ouv. cité, p. 656 et p. 669.

66.. Le Spectateur, 2 octobre 1847.

67.. Idem, 4 décembre 1847.

68.. Charles Forbes MONTALEMBERT, Discours, ouv. cité, p. 663.

69.. Frédéric OZANAM, « Les dangers de Rome et ses espérances », dans Le Correspondant, 1848, pp. 412-435.

70.. Louis LE GUILLOU, Lacordaire-Montalembert, correspondance, 1830-1861, Paris, Éditions du Cerf, 1989, lettre du 19 février 1848.

\section{RÉSUMÉS}

Depuis 1843 , le «mouvement catholique ", avec le comte de Montalembert à sa tête, est entré en lutte avec la Monarchie de Juillet pour la liberté d'enseignement. L'année 1847 apparaît comme un tournant: les événements de Suisse et d'Italie préparent les déchirures de 1848 au sein du mouvement catholique. La correspondance entre Théophile Foisset et Montalembert permet de comprendre mieux cette fracture qui repose sur des présupposés stratégiques, politiques et « culturels ». En janvier 1848, Montalembert prononce deux discours à la Chambre des pairs : l'un sur le Sonderbund, l'autre sur les événements romains. L'étude de la préparation de ces interventions montre les ambiguïtés des positions du chef de file des catholiques libéraux. Visant à la défense des intérêts religieux, ils sont nettement marqués par une prise de position politique. L'orateur, tant par stratégie que par conviction, rend solidaire la religion des intérêts conservateurs de la Monarchie de juillet. Cette confusion semble renier «l'apolitisme » jusque-là invoqué. Ozanam et Lacordaire se désolidariseront. Á la veille des événements de 1848, Montalembert a identifié la défense des intérêts religieux avec un régime qui va disparaître. 
The Catholic movement on the edge of revolution: Switzerland, Italy, France in 1847. Since 1843 , the "Catholic movement ", led by the Comte de Montalembert, had been involved in a long struggle against the July monarchy and in favour of freedom of education. In this respect, the year 1847 stood out as a turning-point: the events in Switzerland and Italy had sown the seeds of dissensions that were eventually to tear apart the Catholic movement, in 1848. The correspondence between Théophile Foisset and Montalembert sheds some light on this rift, brought about by implicit strategic, political and cultural motives. In January 1848, Montalembert delivered two speeches at the Chambre des pairs: one on the Sonderbund, and the other in the events in Rome. A close study of the preparation of these addresses reveals how ambiguous the position adopted by the leader of the Catholic liberals was. Overtly in defence of religious interests, they took up in fact a strong political stance. Both for strategic reasons and out of conviction, the orator bound religion with the conservative interests of the July monarchy. This confusion seemed to repudiate the apolitical attitude put forward up until then. Ozanam and Lacordaire quickly dissociated themselves from this direction. On the eve of the 1848 events, Montalembert strongly identified the defence of religious interests with a political regime close to its end.

\section{AUTEUR}

\section{SYLVAIN MILBACH}

maître de conférence à l'Université de Savoie 